\title{
Reactive sites on the surface of polycyclic aromatic hydrocarbon clusters: A numerical study
}

\author{
Dongping Chen ${ }^{1 *}$ and Kai Hong Luo ${ }^{2}$ \\ ${ }^{1}$ State Key Lab of Explosion Science and Technology, Beijing Institute of \\ Technology, Beijing 100081, China \\ ${ }^{2}$ Department of Mechanical Engineering, University College London, Torrington \\ Place, London, WC1E 7JE, UK \\ ${ }^{*}$ Corresponding Author Email:dc516@bit.edu.cn
}

\begin{abstract}
The surface growth of soot is a key process in its mass growth, depending crucially on surface properties. In this work, we directly extract the detailed surface properties, such as surface area, number density of reactive sites on a particle and parameter $\alpha$, from the microscopic configuration of PAH clusters. Five representative PAHs, including pyrene $\left(\mathrm{C}_{16} \mathrm{H}_{10}\right)$, coronene $\left(\mathrm{C}_{24} \mathrm{H}_{12}\right)$, ovalene $\left(\mathrm{C}_{32} \mathrm{H}_{14}\right)$, hexabenzocoronene $\left(\mathrm{C}_{42} \mathrm{H}_{18}\right)$ and circumcoronene $\left(\mathrm{C}_{54} \mathrm{H}_{18}\right)$, are used to build the model configurations of nascent soot. We develop a numerical scheme to determine the detailed surface properties based on the approximation of solventexcluded surface. The assumption of spherical particles is found to introduce a large uncertainty in the estimation of the surface area, and the error can be a factor of 2.5 in the worst case. The number density of atoms or sites on cluster surfaces does not depend on the chemical composition of a particle larger than $2 \mathrm{~nm}$ in diameter, and our study indicates that the number density of hydrogen atom is overestimated by a factor of 3 compared to a theoretical limit in the literature. Finally, we propose a new equation for parameter $\alpha$, which includes the effects of the size of gaseous species in surface reaction, local temperature, particle size and chemical composition.
\end{abstract}

Keywords: HACA, MD, parameter alpha, solvent-excluded surface, PAH. 


\section{Introduction}

The surface properties of particles, especially nanoparticles, have attracted great attention from both experimentalists [1-6] and modelers [7, 8] due to its importance in the field of nanotechnology. As the particle size approaches to nanoscale, the surface area to volume ratio dramatically increases, which underpins many nanotechnologies. In the catalysis science, the reactivity of catalyst is dependent on the availability of active sites or functional groups which directly interact with reactants on the surface [1]. Besides, in the development of highly active electrocatalysts, the choice of surface representation is critical to the catalytic performance. Experimentally, surface area is usually reported as Brunauer-EmmettTeller (BET) surface area derived from nitrogen adsorption isotherms measured at liquid nitrogen temperature [4]. Recently, Sun et al. commented that the intrinsic activity of electrocatalysts can be artificially misinterpreted using the geometric surface area (i.e. disk surface area), and the surface roughness should be seriously considered [9]. The topology on the surface of a substance is not only related to its reactivity but also its functionality. The high surface area of certain porous materials can provide great capacity for chemical storage and thus enables very promising applications in hydrogen storage [10]. Similarly, in the investigation of biological macromolecules protein-ligand binding systems, the preferred binding site of a ligand on the surface of protein is determined by matching the shape complementarity between the protein and the ligand [6].

Surface reactions play an important role in the mass growth of soot, which have been investigated using various experimental and theoretical approaches [11-18]. A surface reaction involves a gaseous species and an active site on the particle surface [19-21]. However, not all the active sites of a particle are available for surface reactions due to geometric effects [22-24]. This should be taken into account, otherwise the site availability on the surface and thus the rate of surface reaction would be overestimated. Considering the complex nature of soot particles [25], no experimental method is so far capable of quantifying the fraction of inaccessible sites on surface. Instead, Frenklach and Wang [26] proposed a steric 
parameter, $\alpha$, to account for the availability of active sites which can react with gaseous species via the HACA mechanism. In soot models [21, 26, 27], the rate of surface reactions is calculated using the following formula:

$$
R_{s}=\alpha \chi_{i} A_{s} R_{g}
$$

where $R_{S}$ is the rate of surface reaction, $\alpha$ is an empirical parameter accounting for the probability of a gaseous molecule colliding with reactive sites on particle surface, $\chi_{i}$ is the number density of reactive site $i, A_{s}$ is particle surface area, $R_{g}$ is the reaction rate of an analogous gas-phase reaction. This formula was first introduced by Frenklach and Wang [26]. However, there are a few underlying approximations when applying this formula in soot models. Firstly, the parameter $\alpha$ represents a steric phenomenon that should be dependent on the microscopic arrangement of a soot particle. Yet, no one has estimated $\alpha$ numerically due to the lack of a satisfactory microscopic representation. Secondly, similar to the parameter $\alpha, \chi_{i}$ is difficult to estimate from first principle. The current selection assumes that particle surface is covered by PAHs with turbostratic structures. Frenklach and Wang [26] reported $\chi_{H}$ as $2.3 \times 10^{19}$ sites $/ \mathrm{m}^{2}$ from parallel aromatic stacks. This value in fact should be viewed as a theoretical limit of $\chi_{H}$ and no exact value was ever reported afterwards. Thirdly, primary particles of soot are assumed to be spherical particles and thus, surface area is usually reported as a sphericalequivalent area. But in the nano-world, particles do not follow the spherical assumption; for example, mature soot particles [28] usually show complex fractal morphology with a large proportion of overlapping area and more importantly, surface roughness of nanoparticles is an important contributor to surface area, which would be neglected if soot particles are considered as spheres [9]. Last but not the least, activation energy barrier in surface reactions is assumed to be the same as the analogous gas-phase reaction, but this assumption was not verified during the last two decades. These four limitations in equation 1 introduced large uncertainties of the existing soot models. For example, the sensitivity of parameter $\alpha$ was examined in a recent work [29], showing that the selection of parameter $\alpha$ has a 
significant impact on the predicted volume fractions in premixed stretch-stabilized ethylene-oxygen-argon flames. More relevant studies are available in the literature $[27,30,31]$.
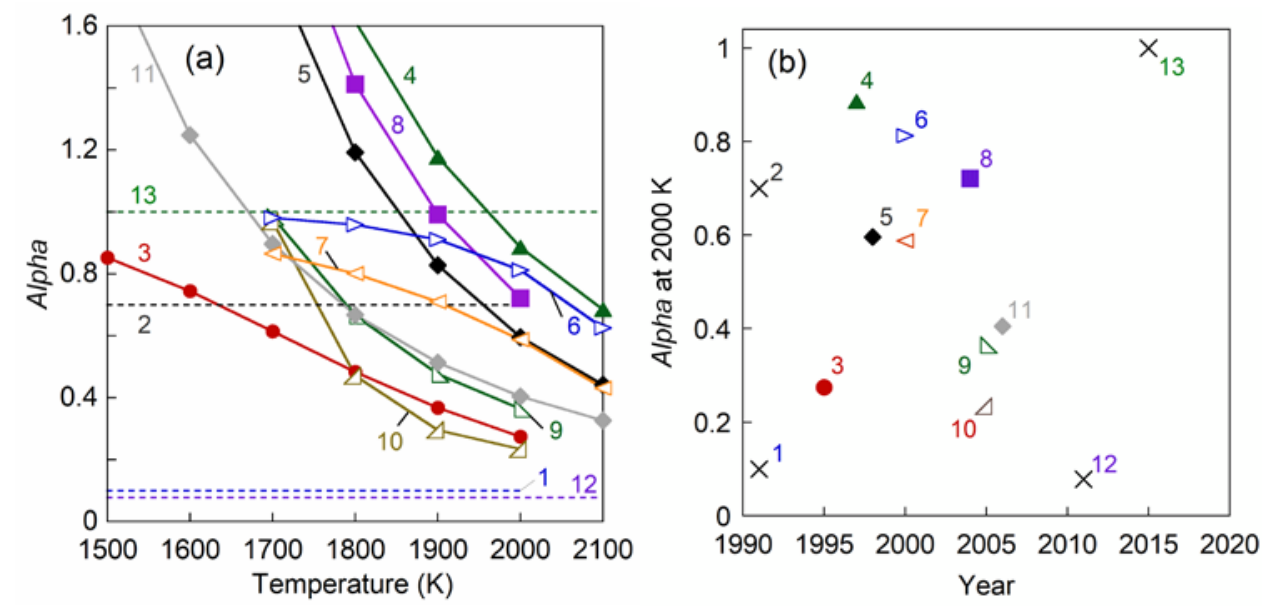

Figure 1. (a) Selected values of parameter $\alpha$ quoted in existing soot models as a function of flame temperature. Solid lines: parameter $\alpha$ is a function of flame temperatures (local or maximum); dashed lines: parameter $\alpha$ is a constant over a specific temperature range. Open symbols (curve 6 and 7) are taken from ABF model with $\mu_{1}=16$ and 100, respectively. (b) The evolution of parameter $\alpha$ from 1991 to 2015 . Cross symbol refers to the cases where parameter $\alpha$ is constant over temperature, while other symbols are consistent with those in Fig. 1a. The references of all curves are listed on Table A1 in the Appendix.

Following Frenklach and Wang's initial investigation, recent studies have further investigated parameter $\alpha$ and use it to quantify surface reactions of soot in laminar premixed flames [13, 26, 32, 33] and non-premixed co-flow/counter-flow flames [34-36]. In these studies, parameter $\alpha$ is found to be a strong function of particle size and flame temperature. For example, Appel et al. [27] proposed an equation that includes both average PAH size and maximum flame temperature to calculate the fraction of reactive surface. In later studies, it is also found that thermal aging causes a reduction in parameter $\alpha$ [31]. Kholghy et al. [37] proposed a surface shell formation model to account for the reactivity difference between nascent soot and 
mature soot primary particles, and captured the change in surface reactivity along with thermal aging. Nascent soot is mainly composed of PAHs without carbonization, while mature soot is carbonized and graphitic as more hydrogen atoms are consumed due to surface growth. Such a structural change from amorphous to more graphitic significantly reduces the surface reactivity. Therefore, the thermal aging effect is critical to revealling surface chemistry for mature soot, while limited effect is seen for nascent soot.

In previous studies of parameter $\alpha$, it was treated as a free parameter when fitted with experiments, while other parameters including $\chi_{i}$ and Arrhenius parameters in $R_{g}$ are considered as constant values. However, this approach causes errors in the fixed parameters to propagate into the fitted parameter $\alpha$, and thus the nature and value of parameter $\alpha$ is buried by errors from other surface parameters in a wide range of experiments in different studies. To illustrate this issue, we extracted values of parameter $\alpha$ in 18 references (Table A1) and plotted $\alpha$ as a function of temperature (local or maximum flame temperature). Overall, 13 correlations are established with 4 constant values and 9 functions of temperature. The range of parameter $\alpha$ shown in Fig. 1a spreads over two orders of magnitude, i.e. 0.078-5.0 for 1500-2100 K. More importantly, no indication of any degree of convergence can be observed during the development from 1991 to 2015 (Fig. 1b). This suggested that each equation of parameter $\alpha$ is only applicable in particular cases, unfortunately, and also highlights the potential large uncertainty in PAH chemistry as its error propagates into the fitted parameter $\alpha$. The experimental measurements of soot volume fraction using optical methods are widely performed to tune parameter $\alpha$, and the scatter of data points in Fig. 1b also suggests that large uncertainty exists in optical properties assumed in the measurements [31]. In comparison, the best experiments give an uncertainty of $15 \%$ in the forward rate of $\mathrm{H}+\mathrm{O}_{2} \rightarrow \mathrm{O}+\mathrm{OH}$ over a wide temperature range of $1100 \mathrm{~K}$ to $3370 \mathrm{~K}$ [38], whereas the uncertainty was a factor of 2 back in 1980 [39]. Unlike the tremendous progress in the study of reaction kinetics of small hydrocarbons chemistry, advancement in the knowledge about surface reactions of soot has been rather incremental. 


\section{In soot community, it is an urgent task to reduce the uncertainties in surface reactions.}

Basically, we cannot only rely on the fitting of experimental data to extract surface properties, where the lack of a satisfactory microscopic representation prohibits us from establishing a solid understanding. The PAH cluster is an appropriate model system for nascent soot as they share similar physical properties such as $\mathrm{C} / \mathrm{H}$ ratio [40], particle density [41], particle size [42], optical properties [43], fringe length and curvature [28]. Recently, Chen and coworkers studied the thermostability of homogenous pyrene and coronene clusters as analogues for nascent soot particles [44] and demonstrated a phase change due to the addition of mass [45]. Also, PAH clusters with larger PAH including ovalene, hexabenzocoronene and circumcoronene were built up in a later work [42]. These studies provided a series of model representations for soot, which enables the theoretical investigation of their surface reactivity. Another preliminary attempt was to probe the surface properties of homogeneous pyrene and coronene clusters using the above representations and an upper bound of parameter $\alpha$ was directly estimated from accessible surface area [46]. Following our previous efforts, in this work, we use homogenous PAH clusters as a model system of nascent soot and examine the surface properties as a function of chemical composition, particle size and temperature. The surface properties include the surface roughness of particles, the number density of reactive sites on PAH clusters and the parameter $\alpha$ in the context of HACA mechanism. Finally, the findings about all the three surface properties are integrated together to illustrate the overall effect on surface reactions.

\section{Computational methods}

The current work studies the surface properties of a number of PAH clusters, $P_{N}$. $N$ denotes the number of molecules, which varies from 50 to 500 in the present study. $P$ represents pyrene (PYR), coronene (COR), ovalene (OVA), hexabenzocoronene (HBC) and circumcoronene (CIR). The detailed molecular structures of these five molecules are shown in Fig. 2. The configurations of these 
clusters are directly taken from previous molecular dynamics (MD) simulations [44, 46]. The morphology of the clusters is either solid-like (parallel PAH stacks) or liquid-like (an irregular configuration), and they were equilibrated at temperatures ranging from 300 to $1300 \mathrm{~K}$. The cluster sizes are in the range of 2.9 to $7.2 \mathrm{~nm}$ (see Table A2 in the Appendix for details). For any particular clusters, 100 different configurations were taken from previous works $[44,46]$. The reported surface properties are computed by averaging over these configurations and the standard deviations are used to present error bars.
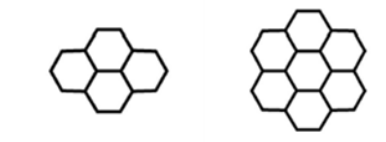

$\operatorname{PYR}\left(\mathrm{C}_{16} \mathrm{H}_{10}\right) \quad$ COR $\left(\mathrm{C}_{24} \mathrm{H}_{12}\right)$

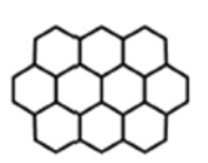

$\operatorname{OVA}\left(\mathrm{C}_{32} \mathrm{H}_{14}\right)$

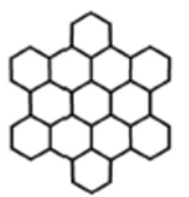

$\operatorname{HBC}\left(\mathrm{C}_{42} \mathrm{H}_{18}\right)$

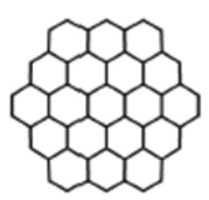

$\operatorname{CIR}\left(\mathrm{C}_{54} \mathrm{H}_{18}\right)$

Figure 2. Molecular structure of five investigated PAHs (pyrene, coronene, ovalene, hexabenzocoronene and circumcoronene).

Solvent-excluded surface (SES) was used to evaluate the actual surface of a cluster, which has been successfully applied in a previous work [46]. The SES area was determined by MSMS 6.2.1 program [47] using a "rolling ball” algorithm. The contributions of each atom on SES area can be directly calculated, and any atom with non-zero contribution is interpreted as a surface atom. This characterisation of the surface atoms forms the basis of this work. The radii of carbon and hydrogen atoms are taken as their van der Waals radii of $1.7 \AA$ and $1.2 \AA$, respectively [48]. To identify site types (Fig. 3) on the surface of microscopic representations, a unique index is assigned to each atom of constituent PAH molecules, such that site type can be extracted via mapping atom indices.

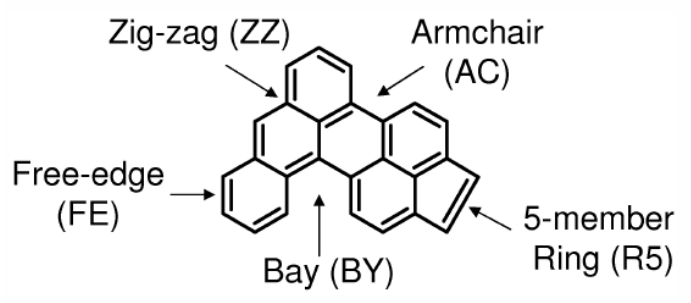


Figure 3. Five possible types of reactive sites on a PAH molecule.

The detailed site information of five representative PAH molecules is listed in Table 1 . The number density of reactive sites (i) on particle surface is defined as:

$$
\chi_{i}=\frac{S_{i}}{A_{S E S}}
$$

where $S_{i}$ and AsEs are the number of reactive site $i$ including $\mathrm{H}$ atom, FE, $\mathrm{ZZ}$ and AC sites on the surface and the solvent excluded surface area accessible to a specific probe, respectively.

Table 1. The number of $\mathrm{H}$ atom, FE, $\mathrm{ZZ}$ and AC sites on different PAH monomers.

\begin{tabular}{ccccc}
\hline Molecule & H atom & FE site & ZZ site & AC site \\
\hline PYR & 10 & 6 & 4 & 0 \\
COR & 12 & 6 & 6 & 0 \\
OVA & 14 & 6 & 8 & 0 \\
HBC & 18 & 12 & 0 & 6 \\
CIR & 18 & 6 & 12 & 0 \\
\hline
\end{tabular}

Parameter $\alpha$ accounts for the steric effect in a collision event between gaseous species and a particle, and it is essentially a probability that the gaseous species collide with a "reactive” site instead of unreactive sites such as basal planes. Thus, parameter $\alpha$ for site $i$ can be computed via the following equation:

$$
\alpha_{i}=\frac{A_{i}}{A_{S E S}}
$$

where $\alpha_{i}$ is parameter $\alpha$ for reactive site $i$ on particle surface, $A_{i}$ and $A_{S E S}$ are the SES area of site $i$ and the total SES area of target configurations.

Considering particle representations in this work, only $\mathrm{H}$ atom can be viewed as a reactive site, i.e. $\alpha_{H}$, and thus, the rest of the paper will focus on $\alpha_{H}$. It is also worth noting that $\alpha_{H}$ is equivalent to $\alpha_{\text {soot }}$ in the context of HACA mechanism as surface reactions start with the abstraction of $\mathrm{H}$ atom from particle surface and forms a corresponding radical (i.e. $C_{\text {soot }}[26]$ ). Physically, $\alpha_{H}$ refers to the 
probability that a $\mathrm{C}_{2} \mathrm{H}_{2}$ molecule collides with a reactive site $\left(C_{\text {soot }}\right.$ ) via addition process. As both $A_{i}$ and $A_{S E S}$ can be estimated using the current numerical scheme, our method herein enables us to estimate important parameter $\alpha_{H}$ for soot or nascent soot for the first time.

\section{Results and discussion}

\subsection{Surface roughness of PAH clusters}

Empirically, the spherical-equivalent surface area $\left(A_{s p h}\right)$ of primary soot particles was reported from the measured particle size assuming a spherical configuration. In our previous study of soot surface [46], we introduced the SES as an alternative representation of the accessible surface. Unlike $A_{s p h}$, SES area (AsES) depends on the size of gaseous species (Fig. 4). In this work, we use a series of spherical "probes" to represent the corresponding gaseous species from 1.2 to $10.0 \AA \AA$; a larger probe would lead to a smoother surface as the probe neglects the local roughness. For the cases of PAH clusters, it is noted that the contribution of hydrogen atom increases with the growth of probe size as $\mathrm{H}$ atoms lie at the edge of the configuration and the large gas molecules always collide on the edge first. Besides, when the probe size is less than $0.5 \AA$, i.e. $r_{P}=0.2 \AA$ (Fig. $4 \mathrm{~A}$ ), the probe penetrates into the particle configuration and particle surface turns to isolated molecular surface. In this case, SES is equivalent to the surface constructed using a volumetric representation of atoms, i.e. $v d w$ representation.

A

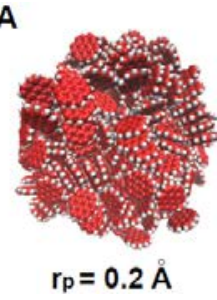

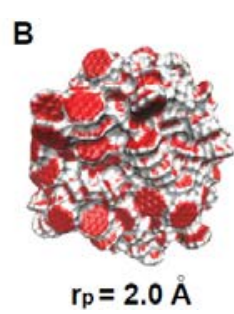

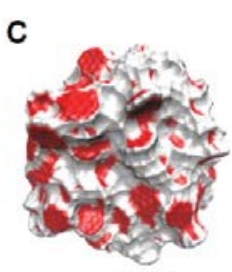

$r p=3.7 \AA$

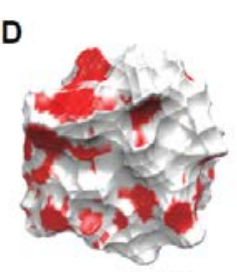

$r p=6.0 \AA$
E

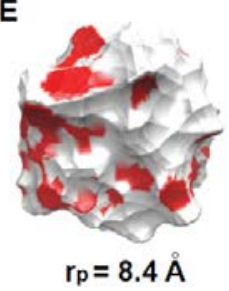

Figure 4. Solvent-excluded surface of a $\mathrm{COR}_{500}$ cluster with probe sizes ranging from 0.2 to $8.4 \AA$. $r_{P}$ represents the size of probe radius. The red and white corresponds to the surface of carbon and hydrogen atoms, respectively. 
The surface roughness is presented as the ratio of $A_{S E S}$ over $A_{\text {sph. }}$. As illustrated in Fig. 5, the surface roughness was plotted as a function of probe size for $\mathrm{COR}_{500}$ and $\mathrm{CIR}_{200}$ clusters with solid or liquid-like configurations. The corresponding diameter for the two representative clusters is 7.2 and $6.5 \mathrm{~nm}$, respectively (Table A1). Four representative molecules, i.e. $\mathrm{C}_{2} \mathrm{H}_{2}$, benzene, $\mathrm{PYR}$, and CIR, as a probe to illustrate the length scale of gaseous species in combustion are highlighted in the figure. The probe size has a significant effect on the estimation of surface roughness. An increase in the probe size can lead to a reduced surface roughness, indicating that particles have a smoother surface when local roughness has been removed. This is consistent with our previous analysis on the surface pocket [46] that a small probe such as $\mathrm{H}$ radical or $\mathrm{C}$ atom could access area beyond the boundary of the particle surface and penetrates into surface pockets, while larger species could not achieve this due to geometric effect. Further investigation reveals that CIR200 clusters show a higher degree of surface roughness by $10 \%$ compared to that of COR500 clusters. The morphology of particle is also a critical factor on the surface roughness and solid configurations have a smaller $A_{S E S} / A_{\text {sph }}$ ratio indicating less surface roughness. For $\mathrm{COR}_{500}$ clusters, the ratio of $A_{S E S} / A_{s p h}$ with probe size of $\mathrm{H}$ radical is 2.23 and 1.50 for configurations at $T_{r}=1.0$ and 0.5 , respectively. Considering a probe as $\mathrm{C}_{2} \mathrm{H}_{2}$, the ratio of AsEs / $A_{\text {sph }}$ is reduced to 1.57 and 1.33. Similar results were extracted when analyzing CIR200 as well, but it is about $10 \%$ larger. The difference between liquid-like and solid configurations vanishes when using larger probes. Further increasing the probe size to $10.0 \AA$, the ratio of $A_{S E S}$ and $A_{\text {sph }}$ converges to 1.15 and 1.20 for $\mathrm{COR}_{500}$ and $\mathrm{CIR}_{200}$, respectively. That means, the spherical approximation on surface area yields at least $15 \%$ error even for a molecule with a radius of 10.0 A. 


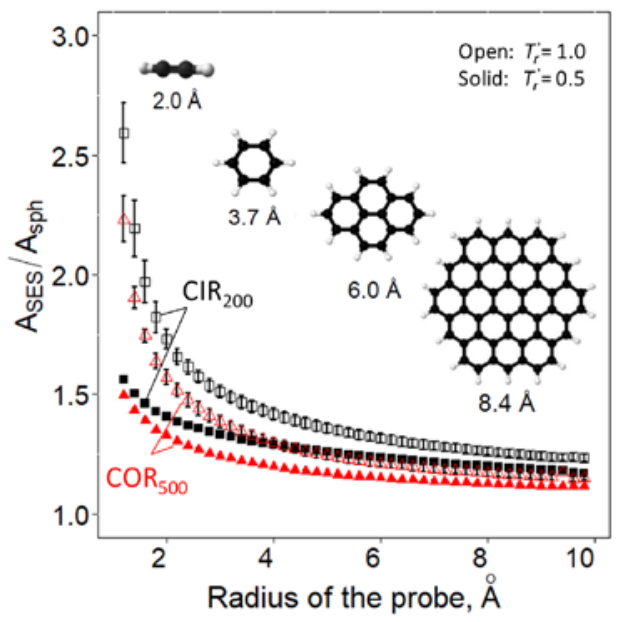

Figure 5. Ratios of SES area $\left(A_{S E S}\right)$ over spherical-equivalent surface area $\left(A_{s p h}\right)$ of $\mathrm{COR}_{500}$ and $\mathrm{CIR}_{200}$ clusters as a function of the probe size. The error bars are standard deviations computed from 100 different configurations. The radius lengths of acetylene, benzene, pyrene and circumcoronene are highlighted in the plot. The data of open and solid symbols are taken from liquid-like configurations at $T_{r}{ }^{\prime}=1.0$ and solid configurations at $T_{r}{ }^{\prime}=0.5$, respectively. $T_{r}{ }^{\prime}$ is defined as $T_{r}{ }^{\prime}=T_{\text {actual }} / T_{c m}$, where $T_{c m}$ refers to the melting point of a cluster.

\subsection{Number density of reactive sites $\left(\chi_{i}\right)$}

The first estimation of $\chi_{H}$ on soot surface was reported in 1991 as $23.0 \times 10^{18}$ sites $/ \mathrm{m}^{2}$ [26]. This value was estimated as two C-H bonds per benzene ring (2.46 $\AA$ ) and the layer separation of benzene stacks was considered as $3.51 \AA$. Similarly, Veshkini et al. [31] calculated $\chi_{H}$ as $14.0 \times 10^{18}$ and $11.5 \times 10^{18} \mathrm{sties} / \mathrm{m}^{2}$ for benzopyrene and coronene stacks, respectively. These values have been widely used in current soot models, but they should be treated as an upper bound of $\chi_{\mathrm{H}}$. Here, we use our numerical scheme to extract the exact $\chi_{H}$ of benzene and coronene stacks. The monomer structures of benzene and coronene are both optimized at B3LYP/6-311+g(d,p) level of theory, and we build stacked monomers with up to 50 monomers, keeping a layer separation of 3.51 A. Figure 6 shows the dependency of calculated $\chi_{H}$ on cluster size, indicating that a larger cluster size yields a higher $\chi_{H}$. The values converge to a constant for clusters with 10 monomers, and the 
corresponding spherical-equivalent diameter is 1.37 and $1.68 \mathrm{~nm}$ for benzene and coronene clusters, respectively. Such an observation can be explained that SES area includes the contributions from both $\mathrm{C}-\mathrm{H}$ bonds on cluster edge and basal planes on the top and bottom of clusters. The contribution from C-H bonds grows linearly when increasing the cluster size, while the surface area of basal planes on the top and bottom of clusters remain constant. The overall effect causes the contribution from $\mathrm{C}-\mathrm{H}$ bonds to dominate SES area so that $\chi_{H}$ converges to a maximum value on large clusters with 50 monomers, i.e. 7.6 and $8.6 \times 10^{18}$ sites $/ \mathrm{m}^{2}$ with a probe size of $2.0 \AA$ for benzene and coronene, respectively. The $\chi H$ of configurations with more than one stack is more representative for a solid particle, and it can be approximated by the values reported in Fig. 6. The geometric effect introduced by multiple stacks is equivalent to hindering the access of certain $\mathrm{C}-\mathrm{H}$ bonds on the cluster edge. However, most of PAHs have a uniform distribution of $\mathrm{C}-\mathrm{H}$ bonds, and thus the overlapping has a limited effect on the overall $\chi_{H}$.

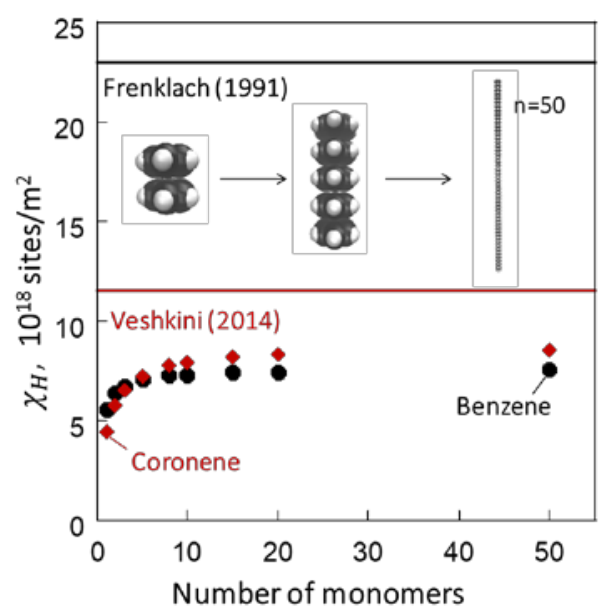

Figure 6. An illustrative estimation of $\chi_{H}$ on stacked benzene and coronene monomers ranging from a monomer to a long stack with 50 monomers. The configurations of benzene clusters are shown on the plot, and the spacing between monomers is kept as $3.51 \AA$. The $\chi_{H}$ is calculated with a probe size of $2.0 \AA$. The literature data proposed by Frenklach and Wang [26] and Veshkini et al. [31] are included as the solid black (benzene) and red lines (coronene), respectively. 
The previous values of $\chi_{H}$ from Frenklach and Wang [26] and Veshkini et al. [31] are of a factor of 3.0 and 1.3 larger than the calculated values for benzene and coronene clusters, respectively (Fig. 6). In the original estimations, surface area was treated as a 2D projection of real surface and all the local roughness was neglected. Thus, the surface area was underestimated and the corresponding $\chi_{H}$ represents a theoretical limit. However, the SES representation in this work enables us to directly estimate the curved surface of particles; the corresponding SES area is much larger compared to the 2D approximation, leading to a smaller $\chi_{H}$. Moreover, our predictions are weakly correlated with the probe size and less than $7 \%$ and $16 \%$ variations are seen when changing the probe size from $1.2 \AA$ to $5.0 \AA$ for benzene and coronene clusters, respectively. Therefore, our approach hereby can be used to provide a better estimate of $\chi_{H}$ for soot modelling and the values from both Frenklach and Wang [26] and Veshkini et al. [31] shall be replaced with our reported values.

Before we report a preferred value for $\chi_{\mathrm{H}}$, let us further examine its size dependence. Fig. 7 illustrates that both $\mathrm{H}$ atoms on the surface and SES area are linearly correlated with the square of particle diameter for all the considered PAHs. The particle size is determined via a rolling ball algorithm and the details can be found in Table A2. Similar correlation can be seen for other surface sites including FE, ZZ and AC sites. These observations are consistent with the physics that any surface property correlates with the square of particle size. More importantly, this indicates an important fact that $\chi_{i}$ is independent of particle size as $\chi_{i}$ equals to the ratio between $S_{i}$ and $A_{S E S}$, and the dependence on the square of particle diameter cancels out. However, considering the detailed parameters of the linear correlation reported in Fig. 7, the current observation is only valid for particles larger than 2 $\mathrm{nm}$ in diameter. Similar observation can be made from Fig. 6 that smaller particles $(<1.5 \mathrm{~nm})$ have a reduced $\chi_{i}$ as the contribution of the basal plane is comparable to that of C-H bonds. 


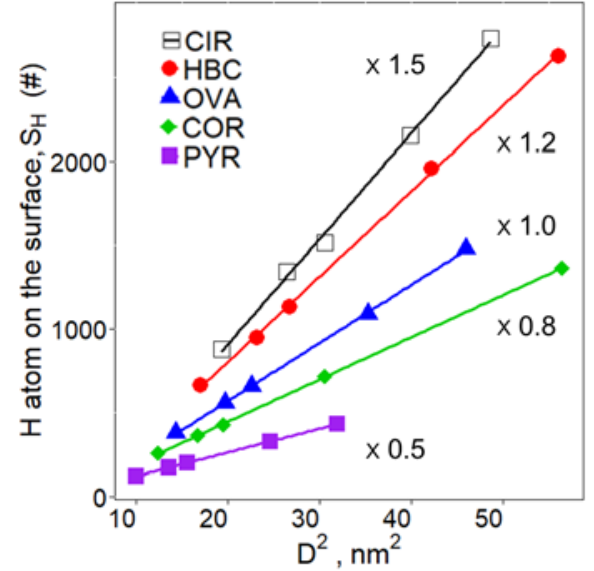

(a) $\mathrm{H}$ atom

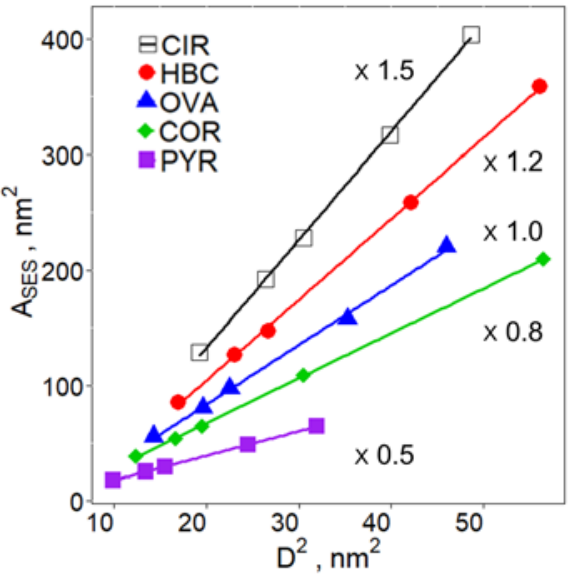

(b) Surface area

Figure 7. The number of $\mathrm{H}$ atoms on particle surface (a) and SES area (b) as a function of the square of particle size at $T_{r}=1.0$. Solid lines are the best fitted lines and the $R^{2}$ of these lines are all above 0.99 . Values on the plots correspond to specific scaling factors applied on each series of data to avoid overlapping. The details of the fitting for both Fig. 7a and $7 \mathrm{~b}$ are listed Table A3 in the Appendix.

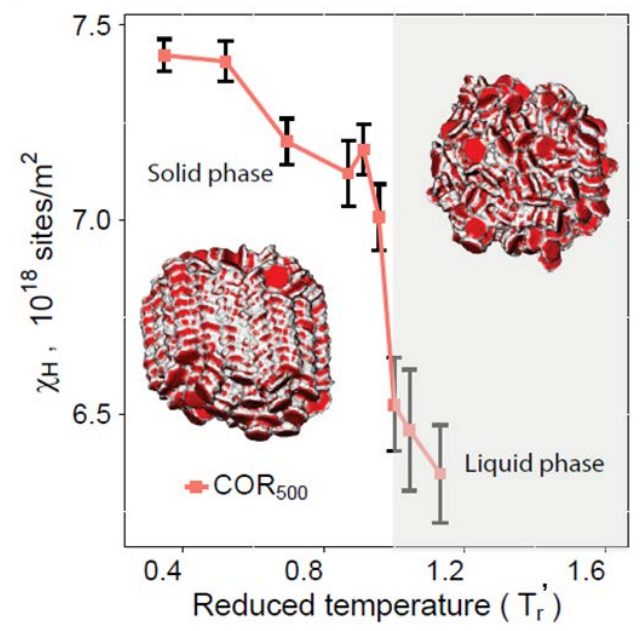

Figure 8. Number density of $\mathrm{H}$ atoms on the surface of a $\mathrm{COR}_{500}$ cluster as a function of the reduced temperature. The radius of probe is $2 \AA$ which is analogous to that of a $\mathrm{C}_{2} \mathrm{H}_{2}$ molecule. The configurations of a COR500 cluster at $T_{r}{ }^{\prime}=0.9$ (left) and 1.0 (right) are also embedded on the plot. The melting point of a COR500 is 575 $\mathrm{K}$ [44]. The error bars are the standard deviations computed from 100 different configurations. 
We then investigated the impact of particle morphology on the exact value of $\chi_{i}$. Here $\chi_{H}$ of $\mathrm{COR}_{500}$ is selected as an example (Fig. 8). Note that this set of data is taken from a previous work of surface availability of $\mathrm{H}$ atoms on COR 500 [46] and replotted as $\chi_{H}$. It is found that the number density of $\mathrm{H}$ atoms on particle surface decreases with the increase of temperature. This is unexpected as surface availability shows an opposite trend. Stacked configurations tend to form smaller stacks and larger surface area at a higher temperature. Thus, the surface availability of a specific atom or site is enhanced. However, considering the $\mathrm{C} / \mathrm{H}$ ratio, more basal carbon atoms become accessible to the gaseous species, and the overall effect yields a smaller $\chi_{H}$ as shown in Fig. 8. We also observe an overall $20 \%$ reduction of $\chi_{H}$ when comparing the value at the minimum temperature $\left(T_{r}{ }^{\prime}=0.4\right)$ with that at the maximum temperature $\left(T_{r}{ }^{\prime}=1.15\right)$. In particular, a $10 \%$ decline in the $\chi_{H}$ is seen when crossing the melting point, and this can be explained by the morphology transition between solid and liquid-like morphology. Once a particle melts, nearly all the distinguished stack configurations break apart, and such a sudden change in the morphology leads to an obvious decrease in $\chi_{H}$. Note that liquid-like morphology may be a representative morphology of soot because the typical flame temperature lies above $1500 \mathrm{~K}$ and particle surface is consistent with irregular stacks [49]. In the following section, only the data of liquid-like morphology will be discussed.

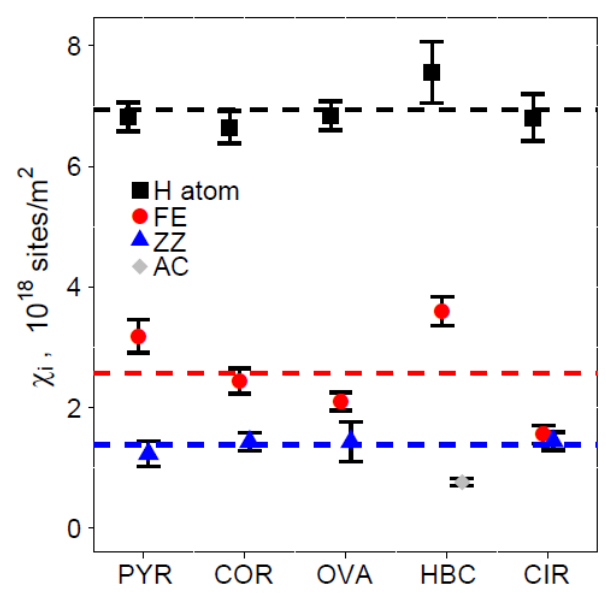


Figure 9. Number density of $\mathrm{H}$ atoms, FE, ZZ and AC sites on the surface of liquidlike PAH clusters at $T_{r}{ }^{\prime}=1.0$. The error bars are the standard deviations computed from 500 different samples of the configurations including data for five cluster sizes. The dashed lines highlight the corresponding average number density across different clusters. The values are computed using $2.0 \AA$ as the probe size and average number density of $\mathrm{H}$ atoms, $\mathrm{FE}$ and $\mathrm{ZZ}$ sites is $7.0 \times 10^{18}, 2.6 \times 10^{18}$ and $1.4 \times 10^{18}$ sites $/ \mathrm{m}^{2}$, respectively.

We further process the data in Fig. 7 and compute $\chi_{i}$ as a function of chemical composition shown in Fig. 9. It is found that $\chi_{i}$ is insensitive to the chemical composition, and the average number density of $\mathrm{H}$ atoms, FE and ZZ sites at $T_{r}{ }^{\prime}=1.0$ is $7.0 \times 10^{18}, 2.6 \times 10^{18}$ and $1.4 \times 10^{18}$ sites $/ \mathrm{m}^{2}$, respectively. However, the spread of $\chi_{F E}$ for different PAHs is wider compared to $\chi_{H}$ and $\chi_{Z Z}$, and the maximum value is about two times that of the minimum. Note that only the HBC molecule has AC sites and the reported value is $1.0 \times 10^{18} \mathrm{sites} / \mathrm{m}^{2}$. The calculated $\chi_{H}$ from different sized PAHs is quite close to the results from the simple approximation as that shown in Fig. 6. As stacked monomers are analogous to solid configurations, we can further take into account the temperature impact as $10 \%$ reduction when comparing with our estimation for liquid-like configurations. The stacked monomers yield $\chi_{H}$ $=6.8 \times 10^{18}$ and $7.7 \times 10^{18}$ sites $/ \mathrm{m}^{2}$ assuming liquid-like configurations for benzene and coronene, respectively. These values are consistent with our estimations in Fig. 9. Thus, stacked monomers can be a potential configuration to estimate the surface properties. Comparing previous $\chi_{H}$, i.e. $23.0 \times 10^{18}$ [26], our computed value is one third of the theoretical limit. Nienow et al. [50] estimated $\chi_{H}$ as $20.1 \times 10^{18} \mathrm{sites} / \mathrm{m}^{2}$ by assuming a hypothetical cubic carbon lattice with density as $1.8 \mathrm{~g} / \mathrm{cm}^{3}$. Their result is close to Frenklach's estimation [26]. The discrepancy between our estimation and their result can be attributed to the inappropriate $2 \mathrm{D}$ approximation of particle surface and also the difference in particle density that is in the range of $1.2-1.4 \mathrm{~g} / \mathrm{cm}^{3}$. More recently, Blanquart and Pitsch [23] used a value of $10.0 \times 10^{18}$ sites $/ \mathrm{m}^{2}$ for $\chi_{H}$ for small particles with a diameter less than $1 \mathrm{~nm}$, while for larger aggregates, they found that $\chi_{H}$ can reach $70.0 \times 10^{18}$ sites $/ \mathrm{m}^{2}$ for a moderate 
temperature at $1630 \mathrm{~K}$. Although we did not estimate $\chi_{H}$ for large aggregates directly, it is known that particle morphology only has moderate impact on $\chi_{H}$ (Fig. 8). Therefore, $\chi_{H}$ of aggregates is unlikely to reach the value suggested by Blanquart and Pitsch [23].

\subsection{Parameter $\alpha$}

From previous works in the literature [26, 27], we learned that parameter $\alpha$ should be a function of chemical composition, and more importantly the temperature of local or maximum flame temperature. As shown in Fig. 10, here for the first time, we illustrate the chemical composition and size dependence of $\alpha_{H}$ considering PAH clusters at $T_{r}{ }^{\prime}=1.0$. The size of included clusters lies in the diameter range of 2.9$7.2 \mathrm{~nm}$, and no dependence of particle size is observed. Similarly, $\alpha_{H}$ is not dependent on the chemical composition as data from different PAH clusters lies in a small range from 0.6 to 0.7 . Therefore, it is concluded that $\alpha_{H}$ is actually insensitive to molecular composition and particle size for all tested PAH clusters at $T_{r}{ }^{\prime}=1.0$; the computed $\alpha_{H}$ is 0.65 on average using a probe with a size similar to acetylene, which is within an upper bound of $\alpha_{H}$ as 0.8-0.9 from our previous work [46].

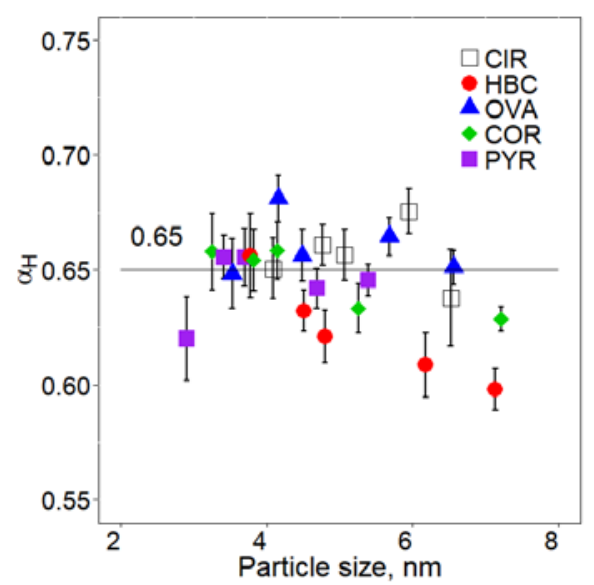

Figure 10. Calculated parameter $\alpha$ for $\mathrm{H}$ atoms on particle surface at $T_{r}{ }^{\prime}=1.0$. The radius of probe is $2 \AA$ which is analogous to that of a $\mathrm{C}_{2} \mathrm{H}_{2}$ molecule. The average value of all data is highlighted by the black line and is shown as 0.65 . 
The above observation can be explained considering the details on particle surface. Since the surface area of active sites is proportional to the number of active sites on particle surface, together with an average surface area that each active site occupies, we can estimate $A_{i}$ via the following equation:

$$
A_{i}=\chi_{i} A_{S E S} A_{i, a v g}
$$

where $A_{i, a v g}$ is the average SES area of reactive site $i$. Following the equations 3 and 4, we can reformulate the calculation of $\alpha_{i}$ as

$$
\alpha_{i}=\chi_{i} A_{i, a v g}
$$

As we learned from the previous section, $\chi_{i}$ has no dependence on either particle size or chemical composition. Table 2 also shows that each carbon or hydrogen atom on the particle surface roughly occupies the same surface area for all the tested clusters. $A_{C, \text { avg }}$ is in the range of 3.77 to $4.25 \AA^{2}$ and 4.09 to $4.38 \AA^{2}$ for solid and liquid-like configurations, respectively. Each hydrogen atom roughly occupies an area of $9.6 \AA^{2}$ for both solid or liquid-like clusters, except $A_{H, a v g}$ on HBC clusters. On average, each of the $\mathrm{H}$ or $\mathrm{C}$ atom on the surface occupies similar surface area, and this is consistent with the physical understanding of particle surface. Thus, $A_{i, a v g}$ including both $A_{C, a v g}$ and $A_{H, a v g}$ does not depend on the particle size or chemical composition. Furthermore, this explains why $\alpha_{H}$ yields a small band as presented in Fig. 10, with the corresponding average value of $\alpha_{H}$ equals to 0.65. For HBC clusters, $A_{H, a v g}$ is about $15 \%$ less than that of other clusters, but $\chi_{H}$ is about $10 \%$ higher. Combining these two factors via equation 5, $\alpha_{H}$ of HBC clusters is about 7\% lower than the average value and lies on the lower bound of all data in Fig. 10. 
Table 2. Average surface area of each carbon and hydrogen atoms on particle surface for both solid and liquid-like configurations.

\begin{tabular}{cccccc}
\hline Surface species & PYR & COR & OVA & HBC & CIR \\
\hline Carbon $\left(\AA^{2}\right)^{\mathrm{a}}$ & $4.25 \pm 0.10$ & $3.77 \pm 0.15$ & $3.77 \pm 0.16$ & $3.76 \pm 0.23$ & $3.74 \pm 0.22$ \\
Hydrogen $\left(\AA^{2}\right)^{\mathrm{a}}$ & $9.54 \pm 0.15$ & $9.79 \pm 0.12$ & $9.71 \pm 0.09$ & $8.32 \pm 0.13$ & $9.65 \pm 0.05$ \\
Carbon $\left(\AA^{2}\right)^{\mathrm{b}}$ & $4.38 \pm 0.09$ & $4.30 \pm 0.15$ & $4.17 \pm 0.11$ & $4.21 \pm 0.14$ & $4.09 \pm 0.11$ \\
Hydrogen $\left(\AA^{2}\right)^{\mathrm{b}}$ & $9.38 \pm 0.13$ & $9.64 \pm 0.06$ & $9.68 \pm 0.07$ & $8.44 \pm 0.05$ & $9.59 \pm 0.05$
\end{tabular}

${ }^{\mathrm{a}}$ Data for solid configurations ranging from $T_{r}{ }^{\prime}=0.4$ to $T_{r}{ }^{\prime}=0.99$.

${ }^{\mathrm{b}}$ Data for liquid-like configurations ranging from $T_{r}{ }^{\prime}=1.0$ to $T_{r}{ }^{\prime}=1.56$.

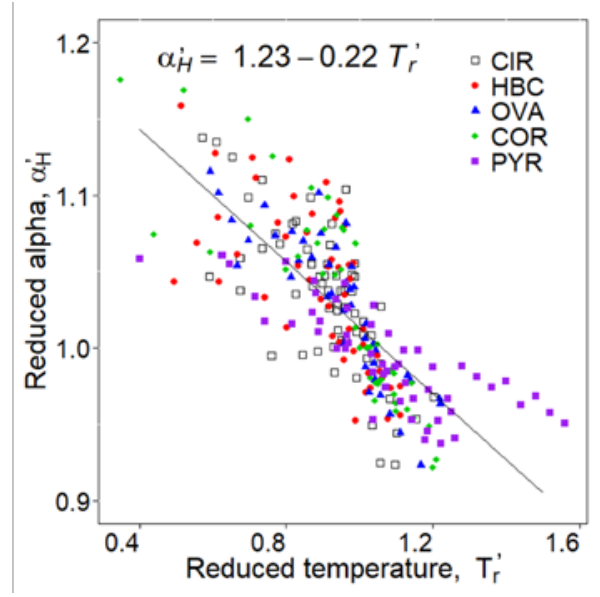

Figure 11. Reduced alpha $\left(\alpha_{H}^{\prime}\right)$ of all PAH clusters ranging from 2.9 to $7.2 \mathrm{~nm}$. The radius of probe is $2 \AA$ and a linear fitted curve is obtained and highlighted in the plot.

A correlation between reduced alpha $\left(\alpha_{H}^{\prime}\right)$ and reduced temperature is presented in Fig. 11. $\alpha_{H}^{\prime}$ is defined as the ratio between $\alpha_{H}$ at a specific temperature and that at $T_{r}{ }^{\prime}=1.0$. Regardless of particle size and molecular composition, it is found that all data clusters together and a linear regression yields a correlation between $\alpha_{H}^{\prime}$ and $T_{r}$ ' as shown in Fig. 11. Interestingly, it is a coincidence that the fitted line almost crosses 1.0 when $T_{r}{ }^{\prime}=1.0$. Moreover, the effects from particle size and chemical composition introduce about $10 \%$ uncertainties on parameter $\alpha$ at the 
same reduced temperature, and this is consistent with the findings in Fig. 10. When considering a specific temperature rather than the reduced one, parameter $\alpha$ is indeed a function of both particle size and chemical composition as melting temperature and thus reduced temperature is correlated with these factors [42, 44]. To explore this matter, we have both $\mathrm{COR}_{500}$ and $\mathrm{CIR}_{200}$ clusters as examples and extrapolate $\alpha_{H}$ at $1800 \mathrm{~K}$. For COR500 clusters, one can convert $1800 \mathrm{~K}$ to $T_{r}{ }^{\prime}=3.13$ according to their melting points (Table A2), and thus the corresponding $\alpha_{H}^{\prime}$ and $\alpha_{H}$ is 0.54 and 0.35, respectively. Similarly, for a CIR200 cluster, $\alpha_{H}$ is 0.59 at $1800 \mathrm{~K}$ as its melting point is $1225 \mathrm{~K}$. Therefore, it is expected that clusters with smaller PAHs always have a lower melting point and the corresponding $\alpha_{H}$ stays away from the average value as shown in Fig. 10 at flame temperatures. Further growth in particle size together with the PAH size would lead that $\alpha_{H}$ slowly approaches to 0.65. In other words, surface reactions accelerate via the increase in parameter $\alpha$. Combining the increase in the surface area, the acceleration should be a non-linear phenomenon if no significant change of configurations occurs along the growth.

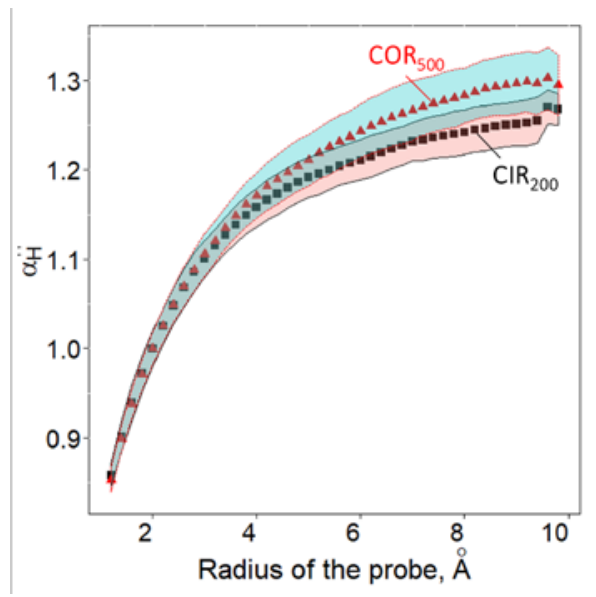

Figure 12. Reduced alpha $\left(\alpha_{H}^{\prime \prime}\right)$ of both $\mathrm{CIR}_{200}$ and COR500 clusters with different probe sizes ranging from $1.2 \AA$ up to $10.0 \AA . \alpha_{H}^{\prime \prime}$ is defined as the ratio between $\alpha_{H}$ computed with a specific probe size over that with a probe size of $2.0 \AA$. The $\alpha_{H}$ of $\mathrm{CIR}_{200}$ and $\mathrm{COR}_{500}$ with $r_{p}=2.0 \AA$ is 0.67 and 0.62 , respectively. The shaded area indicates the standard deviation calculated from 100 configurations. 
The impact of probe size is addressed as a final factor here. As previously discussed, all surface properties including surface area and the number density of active sites is subject to the type of gaseous species, or more precisely, the probe size. The dependence of probe size of $\alpha_{H}$ is presented in Fig. 12 for CIR 200 and COR500. To clearly identify the impact of probe size on $\alpha_{H}$, we further define a reduced $\alpha_{H}^{\prime \prime}$ as the ratio between $\alpha_{H}$ computed with a specific probe size over that with a probe size of $2.0 \AA$. When probe size is smaller than $4.0 \AA$, the two cases agree with each other but further increase in the probe size makes the predicted alpha values deviate. This may correlate with the porosity of the particle surface and $\mathrm{COR}_{500}$ has a higher amount of holes or pockets [46] on particle surface. It is worth noting that the smallest probe here is $1.2 \AA$ representing an $\mathrm{H}$ radical and its alpha value is about $15 \%$ smaller than that of $\mathrm{C}_{2} \mathrm{H}_{2}$.

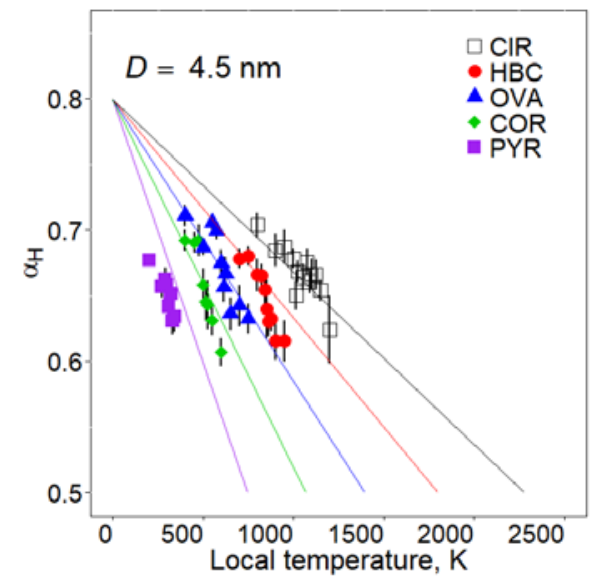

Figure 13. A new equation of parameter $\alpha$ proposed in this work. The points are taken from MD simulations with a particle diameter of about $4.5 \mathrm{~nm}$, i.e. PYR200, $\mathrm{COR}_{100}$, OVA100, HBC80, and CIR80, while the lines are presented via equation 6.

Combining previous works on melting points of PAH clusters [42, 44], we establish a new equation to estimate parameter $\alpha_{H}$ for single primary particle :

$$
\alpha_{H}=A_{H}\left[1.23-\frac{0.22 T}{\left(1-\frac{1.22}{D}\right)(52.01+2.16 m)}\right],
$$


where $A_{H}$ is the value of parameter $\alpha_{H}$ at $T_{r}{ }^{\prime}=1.0, T$ is the local temperature or particle temperature in $K, D$ is the particle spherical diameter in $n m$, and $m$ is the molecular mass of PAHs in a.u.. Note that one should take into account the size of gaseous species in $A_{H}$ and the corresponding effect is shown in Fig. 12. The detailed derivation can be found in the Appendix. As shown in Fig. 13, the proposed equation well captures the data of clusters with a size about $4.5 \mathrm{~nm}$. Comparing with previous works shown in Fig 1., it is noted that the predicted parameter $\alpha$ from equation 6 is much smaller than the previous values. At $2000 \mathrm{~K}$, parameter $\alpha$ is 0.24 and 0.54 for a COR and CIR particle with a size as $4.5 \mathrm{~nm}$, respectively, while ABF model [27] yields the corresponding values as 0.76 and 0.65 . In a recent work, Frenklach [30] revamped the treatment of parameter $\alpha$ that correlates with the carbon networking in the particle structure, but the initial reactive state of particle surface was extracted from a fitting of experimental data. In previous works [31, 37], parameter $\alpha$ commonly decreases with increasing particle size or its thermal age in flames, however, equation 6 here predicts an opposite trend. We speculate that such contradiction might arise from our selected model systems, i.e. PAH clusters, and they fail to represent the configurations of mature soot owing to the lack of carbonization [23, 27]. An appropriate model system should be considered in future work to address the reactivity of mature soot. Another limitation of equation 6 is that the derivation is based on an isolated particle and its validity for aggregates is subject to further investigation. Following the SES representation of particle surface, we explicitly account for the effect of particle morphology as solid and liquid-like configurations using microscopic representations of nascent soot and provide a physical insight into surface properties. It is also important to note that the size effect of gas species is introduced for the first time. The new proposed correlation is critical for the future development of soot models to capture the physics in surface reactions of nascent soot.

\subsection{Overall effect}


Table 3. Comparison between literature values and findings in the current work from $500 \mathrm{~K}$ to $2000 \mathrm{~K}$.

\begin{tabular}{ccccccccc}
\hline \multirow{2}{*}{ Parameters $^{\mathrm{a}}$} & \multicolumn{2}{c}{$500 \mathrm{~K}$} & \multicolumn{2}{c}{$1000 \mathrm{~K}$} & \multicolumn{2}{c}{$1500 \mathrm{~K}$} & \multicolumn{2}{c}{$2000 \mathrm{~K}$} \\
\cline { 2 - 8 } & COR & CIR & COR & CIR & COR & CIR & COR & CIR \\
\hline $\begin{array}{c}\text { Normalized } \\
\text { surface } \\
\text { area }^{\mathrm{b}}\end{array}$ & 1.57 & 1.73 & 1.57 & 1.73 & 1.57 & 1.73 & 1.57 & 1.73 \\
$\begin{array}{c}\text { Normalized } \\
\chi_{H}{ }^{c}\end{array}$ & 0.30 & 0.36 & 0.30 & 0.36 & 0.30 & 0.30 & 0.30 & 0.30 \\
$\begin{array}{c}\text { Normalized } \\
\alpha_{H}{ }^{\mathrm{d}}\end{array}$ & 0.66 & 0.73 & 0.52 & 0.67 & 0.38 & 0.62 & 0.32 & 0.82 \\
$\begin{array}{c}\text { Overall } \\
\text { effect }\end{array}$ & 0.31 & 0.45 & 0.24 & 0.42 & 0.18 & 0.32 & 0.15 & 0.43 \\
\hline
\end{tabular}

${ }^{\mathrm{a}} \mathrm{A} \mathrm{C}_{2} \mathrm{H}_{2}$ molecule is considered as the probe, i.e. $2 \AA$.

b The normalized surface area is defined as the ratio of $A_{S E S}$ and $A_{s p h}$, and data listed here is taken from Fig. 5 for $T_{r}=1.0$.

${ }^{\mathrm{c}} \chi_{H}$ is normalized by a value of $23.0 \times 10^{18}$ from [26], and data listed here is taken for $T_{r}=1.0$. The temperature effect is considered as $20 \%$ reduction for the transition from a solid to liquid-like configuration.

${ }^{\mathrm{d}}$ Parameter $\alpha_{H}$ here is first calculated from equation 6 assuming $D=4.5 \mathrm{~nm}$. Then, the values are all normalized by data taken from ABF model [27] with $\mu_{1}=24$ and 54 for COR and CIR clusters, respectively.

A detailed comparison between literature values widely used in the past and our current findings is shown in Table 3, and all reported values are normalized to better illustrate the difference. Two representative molecules, i.e. COR and CIR, are considered and the corresponding parameters were analyzed at four temperatures, i.e. $500 \mathrm{~K}, 1000 \mathrm{~K}, 1500 \mathrm{~K}$ and $2000 \mathrm{~K}$, to cover a wide range of temperature effect. As shown in Table 3, all the three parameters including surface area, $\chi_{H}$ and $\alpha_{H}$ have a significant difference between literature values and our findings. The 
previous studies underestimate the surface area by 50-70\% while both $\chi_{H}$ and $\alpha_{H}$ are overestimated by a factor of 3 at maximum. Besides temperature, $\alpha_{H}$ for both previous approach and our proposed correlation (equation 6) depends on the molecular composition, and the underlying reason is previously discussed as the difference in the melting temperature. We further consider particle size as an independent term to describe its impact on the melting temperature as well. The size effect of gaseous species involving in surface reactions is explicitly included in our approach (equation 6). Overall, taking all the three terms together via equation 1, the empirical approach overestimates the contribution of surface properties on surface reactions, and the discrepancy can be as large as a factor of 6 and 3 for COR and CIR clusters, respectively. Furthermore, we can question the previous estimation of $R_{g}$ as the errors in the $R_{g}$ actually propagate into the surface parameters, especially parameter $\alpha$ as we discussed in Introduction. In other words, the Arrhenius parameters in the expression of $R_{g}$ overestimate the energy barrier for surface reactions; for example, considering COR clusters at $2000 \mathrm{~K}$ and no change in the parameter $A$ and $n$, one can estimate the difference in the energy barrier between surface reaction and gas-phase reaction as $7.2 \mathrm{kcal} / \mathrm{mol}$. This reduced energy barrier can be attributed to the interactions of molecules on particle surface; however, the detailed mechanism is worth further investigation in future.

The current analysis of surface properties examines the geometric effect only, neglecting the interactions between the probe and particle surface. This approximation may not hold for the area located in a surface pocket that suffers higher energy barrier to access. Also, homogeneous PAH clusters might not be a good model for late stages of soot surface growth. Nevertheless, it is the first time that the surface properties of PAH clusters are investigated via a series of physical representations of particles. When considering the findings presented in this work, we shall not ignore the uncertainty in the surface properties, and further studies shall continue the investigation into the correlation between surface properties and clusters built from molecules such as planar PAHs with substitutions [51, 52], cross-linked PAHs [53-56], oxygenated PAHs [52] and even curved PAHs [57, 58]. 
It is expected that soot particles covered with latter three types of PAHs follow the same trends in the surface properties of homogeneous PAHs as they also prefer stacked configuration. In comparison, the functional groups on surface PAHs would impact the surface dynamics due to the limited accessibility of active site on particle surface. Instead, the surface reaction is more likely to occur on the functional groups.

\section{Conclusions}

The surface properties of PAH clusters including surface roughness, number density of reactive sites on particle surface and parameter $\alpha$ are carefully investigated using SES representation. The effects on each surface property from four independent parameters, i.e. the size of gaseous species, temperature, particle size and chemical composition, are examined. A small species could access area beyond the boundary of the particle surface, particularly surface pockets, and lead to a large accessible area on particle surface. Besides, both chemical composition and temperature have a strong effect on the prediction of surface area for a small species ( $<4 \AA$ ). It is also found that the assumption of spherical particles introduces a large uncertainty in the estimation of the surface area, and in the worst case, the error can be as large as a factor of 2.5. It is worth noting that the surface accessibility of reactive sites decreases with the increase in temperature. The number density of atoms or sites on cluster surface does not depend on the chemical composition of a particle, and this finding is consistent with the physical understanding of particle surface. The number density of $\mathrm{H}$ atoms on particle surface estimated from our method suggests that the value is overestimated by a factor of 3 in the literature.

Furthermore, the parameter $\alpha$, which is usually treated as a fitting parameter in the models, has been systematically analyzed using structures analogous to nascent soot for the first time. Surprisingly, parameter $\alpha$ for $\mathrm{H}$ abstraction reactions is insensitive to the chemical composition in the particle and no particle size dependence is observed at the same reduced temperature. On the other hand, temperature has an obvious impact on parameter $\alpha$ and we quantify its impact using a linear correlation over a wide range of temperatures. The size of gaseous species 
has a noticeable impact on parameter $\alpha$, and this should be taken into account when estimating parameter $\alpha$ for a specific reaction. More importantly, a new equation for parameter $\alpha$ is proposed and its derivation is mainly based on the extrapolation of current data.

The above findings have also been discussed in the context of energy barrier in surface reactions, and we conclude that the activation energy is lower than that of gaseous reaction by $7.2 \mathrm{kcal} / \mathrm{mol}$ at $2000 \mathrm{~K}$ and this is based on the overall impact on the three surface parameters. Therefore, we believe our new findings are valuable for the development of a proper surface model for soot, and should be further integrated into a soot model and guide the parameter selection for surface reactions of nascent soot at least.

\section{Acknowledgements}

This work was supported by the National Natural Science Foundation of China (No. 51806016) and Beijing Institute of Technology Research Fund Program for Young Scholars. Support from the UK Engineering and Physical Sciences Research Council under the project "UK Consortium on Mesoscale Engineering Sciences (UKCOMES)” (Grant No. EP/R029598/1) is gratefully acknowledged. 


\section{Appendix}

1. Parameter $\alpha$ in the literature

Table A1. Detailed descriptions of parameter $\alpha$ in the literature.

\begin{tabular}{|c|c|c|}
\hline References & Model function or constant & Index in Fig. 1 \\
\hline Frenklach and Wang (1991) [26] & 0.1 & 1 \\
\hline Frenklach and Wang (1991) [26] & 0.7 & 2 \\
\hline Kazakov et al. (1995) [13] & {$\left[\tanh \left(8168 / T_{\max }-4.57\right)+1\right] / 2$} & 3 \\
\hline Xu et al. (1997) [32] & & \multirow[b]{2}{*}{4} \\
\hline $\begin{array}{l}\text { Zhang et al. (2009) [59] } \\
\text { Liu et al. (2012) [60] }\end{array}$ & $0.004 \exp (10800 / T)^{\mathrm{a}}$ & \\
\hline \multicolumn{3}{|l|}{ Saffaripour et al.(2011) [61] } \\
\hline Xu et al. (1998) [33] & $0.00115 \exp (12500 / T)^{\mathrm{a}}$ & 5 \\
\hline Appel et al. (2000) [27] & $\tanh \left(a / \log \mu_{1}+b\right)^{\mathrm{b}}$ & $\begin{array}{l}6\left(\mu_{1}=16\right) \\
7\left(\mu_{1}=100\right)\end{array}$ \\
\hline \multicolumn{3}{|l|}{ El-Leathy et al. (2004) [62] } \\
\hline Kim et al. (2004) [14] & $0.0017 \exp (12100 / T)^{\mathrm{a}}$ & 8 \\
\hline \multicolumn{3}{|l|}{ Zhao et al. (2017) [63] } \\
\hline Singh et al. (2005) [64] & $\begin{array}{c}1 \text { for } A_{p} \leq 0.012^{\mathrm{c}} \\
0.2 \text { for } A_{p}>0.012^{\mathrm{c}}\end{array}$ & - \\
\hline Singh et al. (2005) [64] & $0.2+0.8 \exp \left(\left(882-0.52 T_{\max }\right) A_{p}\right)$ & $\begin{array}{l}9\left(A_{p}=0.01 \mathrm{~s}\right) \\
10\left(A_{p}=0.02 \mathrm{~s}\right)\end{array}$ \\
\hline Guo et al. (2006) [65] & $0.0045 \exp (9000 / T)^{\mathrm{a}}$ & 11 \\
\hline Dworkin (2011) [66] & 0.078 & 12 \\
\hline Veshkini et al. (2014) [31] & $6974.6 / T_{a}^{2} \exp \left(-88.06 / T_{a}\right)^{\mathrm{d}}$ & - \\
\hline Wang et al. (2015) [67] & 1.0 & 13 \\
\hline
\end{tabular}


Khosousi and Dworkin (2015) [68] $\quad\left(T_{a, \max } / T_{a}\right)^{2.2} \exp \left[\left(2.4^{*}\left(0.85-T_{a, \max } / T_{a}\right)\right]^{\mathrm{e}}\right.$

Khosousi and Dworkin (2015) [69] $\quad\left(T_{a, \max } / T_{a}\right)^{2} \exp \left[\left(2 *\left(1-T_{a, \max } / T_{a}\right)\right]^{\mathrm{e}}\right.$

${ }^{a} T$ is local temperature.

${ }^{\mathrm{b}} \mu_{1}$ is the first reduced moment of the particle size distribution representing the average mass per particle. $a$ and $b$ are fitted parameters that follows $12.56-0.00563$ $T_{\max }$, and $-1.38+0.00068 T_{\max }$, respectively, and $T_{\max }$ refers to the maximum flame temperature.

${ }^{c} A_{p}$ is particle residence time in second.

${ }^{\mathrm{d}} T_{a}$ is the thermal age and is defined as the integral of temperature to which a particle has been exposed with respect to time.

e $T_{a, \max }$ refers to the thermal age at the point of maximum soot. 
2. Particle properties

Table A2. Featured properties of all clusters at the melting point and melting point temperatures

\begin{tabular}{|c|c|c|c|c|}
\hline Cluster $^{\mathrm{a}}$ & $\begin{array}{l}\text { Spherical- } \\
\text { equivalent diameter } \\
\text { in this work }(\mathrm{nm})^{\mathrm{b}}\end{array}$ & $\begin{array}{c}\text { Spherical-equivalent } \\
\text { diameter in previous } \\
\text { works }(\mathrm{nm})^{\mathrm{c}}\end{array}$ & $\begin{array}{c}\text { SES area } \\
\left(\mathrm{nm}^{2}\right)\end{array}$ & $\begin{array}{l}\text { Melting } \\
\text { point (K) }\end{array}$ \\
\hline $\mathrm{PYR}_{50}$ & 2.91 & 3.16 & $34.39 \pm 0.56$ & 250 \\
\hline $\mathrm{PYR}_{80}$ & 3.41 & 3.68 & $48.53 \pm 0.77$ & 270 \\
\hline PYR $_{100}$ & 3.70 & 3.94 & $55.93 \pm 0.76$ & 280 \\
\hline $\mathrm{PYR}_{200}$ & 4.69 & 4.96 & $91.68 \pm 1.14$ & 310 \\
\hline $\mathrm{PYR}_{300}$ & 5.39 & 5.65 & $122.09 \pm 1.85$ & 320 \\
\hline $\mathrm{COR}_{50}$ & 3.26 & 3.52 & $45.45 \pm 0.80$ & 455 \\
\hline $\mathrm{COR}_{80}$ & 3.83 & 4.09 & $63.11 \pm 1.57$ & 480 \\
\hline $\mathrm{COR}_{100}$ & 4.51 & 4.42 & $75.10 \pm 1.54$ & 500 \\
\hline $\mathrm{COR}_{200}$ & 5.26 & 5.53 & $128.13 \pm 1.44$ & 525 \\
\hline $\mathrm{COR}_{500}$ & 7.21 & 7.51 & $251.25 \pm 5.55$ & 575 \\
\hline $\mathrm{OVA}_{50}$ & 3.53 & 3.78 & $54.68 \pm 1.07$ & 575 \\
\hline $\mathrm{OVA}_{80}$ & 4.16 & 4.44 & $77.24 \pm 1.72$ & 600 \\
\hline $\mathrm{OVA}_{100}$ & 4.49 & 4.75 & $92.56 \pm 1.79$ & 615 \\
\hline $\mathrm{OVA}_{200}$ & 5.68 & 5.94 & $151.63 \pm 3.27$ & 650 \\
\hline $\mathrm{OVA}_{300}$ & 6.56 & 6.78 & $209.10 \pm 3.62$ & 675 \\
\hline $\mathrm{HBC}_{50}$ & 3.78 & 4.11 & $68.77 \pm 1.95$ & 810 \\
\hline $\mathrm{HBC}_{80}$ & 4.51 & 4.81 & $102.46 \pm 2.35$ & 875 \\
\hline $\mathrm{HBC}_{100}$ & 4.80 & 5.17 & $117.73 \pm 3.16$ & 900 \\
\hline $\mathrm{HBC}_{200}$ & 6.18 & 6.49 & $214.67 \pm 8.13$ & 975 \\
\hline
\end{tabular}




\begin{tabular}{ccccc}
\hline $\mathrm{HBC}_{300}$ & 7.13 & 7.49 & $290.29 \pm 8.73$ & 990 \\
$\mathrm{CIR}_{50}$ & 4.09 & 4.39 & $83.49 \pm 2.99$ & 1040 \\
$\mathrm{CIR}_{80}$ & 4.77 & 5.14 & $124.63 \pm 3.94$ & 1090 \\
$\mathrm{CIR}_{100}$ & 5.16 & 5.52 & $138.95 \pm 6.50$ & 1150 \\
$\mathrm{CIR}_{150}$ & 5.95 & 6.31 & $194.02 \pm 4.75$ & 1185 \\
$\mathrm{CIR}_{200}$ & 6.52 & 6.97 & $236.29 \pm 7.26$ & 1225 \\
\hline
\end{tabular}

${ }^{\mathrm{a}}$ Indices refer to the number of molecules in the cluster. Data of PYR, COR are taken from Ref. [44], and data of OVA, HBC and CIR are taken from Ref. [42].

b In this work, we calculate spherical-equivalent diameters from a rolling ball algorithm and assume a spherical particle. The probe size is $2.0 \AA$.

c Spherical-equivalent diameters are taken from previous work [42, 44] that were estimated from a Monte Carlo algorithm [41].

In this work, smaller spherical-equivalent diameters are reported compared to the values in our previous works $[42,44]$. The discrepancy is caused by the change in computing algorithm. We compute the cluster volume using a rolling ball algorithm that generates a connected particle surface this time, while a Monte Carlo (MC) algorithm was previously used to compute the cluster volume and a scaling factor, i.e. 1.7 [41], was included to encompass all intermolecular space by scaling the size of individual carbon and hydrogen atom. The application of scaling factor highlights the issue in MC algorithm for volume calculations, as volumetric representation cannot represent the particle boundary very well and particle surface is isolated. In contrast, rolling ball algorithm precisely identifies the particle surface and thus yields a smaller and more accurate diameter.

\section{Parameters in the fitting of Fig. 7}

Table A3. Detailed parameters in the fitting of both Fig. 7a and 7b.

\begin{tabular}{ccccc}
\hline \multirow{2}{*}{ Cluster } & \multicolumn{2}{c}{$\begin{array}{c}\text { H atom on surface, } \\
\mathrm{S}_{\mathrm{H}}(\#)^{\mathrm{a}}\end{array}$} & \multicolumn{2}{c}{$\begin{array}{c}\text { SES area, } \\
\text { AsES }\left(\mathrm{nm}^{2}\right)^{\mathrm{b}}\end{array}$} \\
\cline { 2 - 5 } & $\mathrm{A}$ & $\mathrm{B}$ & $\mathrm{A}^{\prime}$ & B $^{\prime}$ \\
\hline PYR & -33.4 & 28.1 & -6.4 & 4.2 \\
\hline
\end{tabular}




\begin{tabular}{ccccc}
\hline COR & -70.9 & 31.5 & -13.5 & 4.9 \\
OVA & -121.0 & 34.7 & -20.3 & 5.2 \\
HBC & -180.0 & 42.5 & -30.6 & 5.9 \\
CIR & -233.0 & 42.0 & -37.3 & 6.3 \\
\hline
\end{tabular}

${ }^{\text {a }} \mathrm{S}_{\mathrm{H}}=\mathrm{A}+\mathrm{B} \bar{D}^{2}$ and $D$ represents the spherical diameter of a cluster.

${ }^{\text {b }}$ AsES $=A^{\prime}+B^{\prime} D^{2}$ and $D$ represents the spherical diameter of a cluster.

4. Derivation for equation 6

Regardless of chemical composition and particle size, parameter $\alpha$ at $T_{r}{ }^{\prime}=1.0$ yields an average of 0.65 for a reaction involving species with a radius of $2.0 \AA$. Further analysis on the temperature impact (Fig. 11) reveals that parameter $\alpha$ can be represented via a fitted equation:

$$
\alpha=0.65\left(1.23-0.22 T_{r}^{\prime}\right) .
$$

This equation can be generalized to describe parameter $\alpha$ for any sized gaseous species with a parameter $A_{H}\left(=0.65 \alpha_{H}^{\prime \prime}\right)$. The key point is to estimate $T_{r}$, which can be rewritten as

$$
T_{r}^{\prime}=\frac{T}{T_{c m}}=\frac{T}{T_{r} \cdot T_{b u l k}}
$$

where $T_{r}=T_{c m} / T_{b u l k}$ and $T_{b u l k}$ refers to a bulk melting point of a species. From previous works, it was learned that $T_{r}$ is linearly correlated with the inverse of particle diameter as $T_{r}=1-b / D$, where $b$ is a parameter for different PAHs ranging from 0.95 to $1.45[42,44]$. In this work, we refitted $T_{r}$ as a function of the inverse of particle diameter to remove the dependence on chemical composition, as shown in Fig. A1. Tbulk can be calculated from the molecular mass of a species and the correlation is taken from Fig. 4 [42] as

$$
T_{\text {bulk }}=52.01+2.16 m \text {, }
$$

Where $m$ is the molecular mass in a.u.. Combing the above equations, parameter $\alpha$ can be calculated via the following equation:

$$
\alpha_{H}=A_{H}\left[1.23-\frac{0.22 T}{\left(1-\frac{1.22}{D}\right)(52.01+2.16 m)}\right]
$$




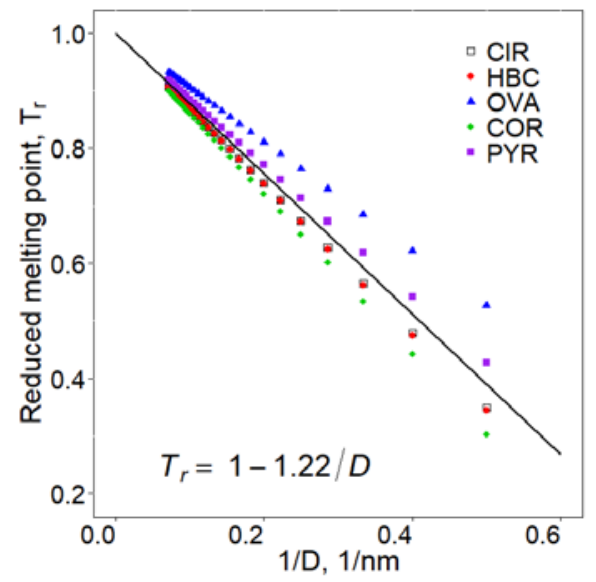

Figure A1. Refitted reduced melting point $\left(T_{r}\right)$ as a function of the inverse of particle diameter. $T_{r}$ is defined as $T_{r}=T_{c m} / T_{b u l k}$, where $T_{c m}$ and $T_{b u l k}$ refer to the melting point of a cluster and a bulk melting point, respectively. The points are taken from the correlation shown in Fig. 3 [42] and Fig. 5 [44]. The black line is refitted by averaging $T_{r}$ at same particle size.

\section{References}

[1] P. Chen, X. Zhou, N. Andoy, K. Han, E. Choudhary, N. Zou, G. Chen, H. Shen, Spatiotemporal catalytic dynamics within single nanocatalysts revealed by singlemolecule microscopy, Chemical Society Reviews 43 (2014) 1107-1117.

[2] J. Dupont, J. Scholten, On the structural and surface properties of transitionmetal nanoparticles in ionic liquids, Chemical Society Reviews 39 (2010) 17801804.

[3] A.K. Gupta, M. Gupta, Synthesis and surface engineering of iron oxide nanoparticles for biomedical applications, Biomaterials 26 (2005) 3995-4021.

[4] H.W. Langmi, A. Walton, M.M. Al-Mamouri, S.R. Johnson, D. Book, J.D. Speight, P.P. Edwards, I. Gameson, P.A. Anderson, I.R. Harris, Hydrogen adsorption in zeolites A, X, Y and RHO, Journal of Alloys and Compounds 356357 (2003) 710-715.

[5] Y. Lu, Y. Yin, B.T. Mayers, Y. Xia, Modifying the surface properties of superparamagnetic iron oxide nanoparticles through A sol-gel approach, Nano Letters 2 (2002) 183-186.

[6] S. Shoichet, I.Kuntz, D. Bodian, Molecular docking using shape descriptors, Journal of Computational Chemistry 13 (1992) 380-397.

[7] T.X. Sayle, M. Molinari, S. Das, U.M. Bhatta, G. Mobus, S.C. Parker, S. Seal, D.C. Sayle, Environment-mediated structure, surface redox activity and reactivity of ceria nanoparticles, Nanoscale 5 (2013) 6063-6073. 
[8] H.C. Schwarzer, W. Peukert, Prediction of aggregation kinetics based on surface properties of nanoparticles, Chemical Engineering Science 60 (2005) 11-25.

[9] S. Sun, H. Li, Z. Xu, Impact of surface area in evaluation of catalyst activity, Joule 2 (2018) 1024-1027.

[10] H. Frost, T. Düren, R.Q. Snurr, Effects of surface area, free Volume, and heat of adsorption on hydrogen uptake in metal-organic frameworks, The Journal of Physical Chemistry B 110 (2006) 9565-9570.

[11] M. Balthasar, F. Mauss, A. Knobel, M. Kraft, Detailed modeling of soot formation in a partially stirred plug flow reactor, Combustion and Flame 128 (2002) 395-409.

[12] A. D'Anna, J.H. Kent, A model of particulate and species formation applied to laminar, nonpremixed flames for three aliphatic-hydrocarbon fuels, Combustion and Flame 152 (2008) 573-587.

[13] A. Kazakov, H. Wang, M. Frenklach, Detailed modeling of soot formation in laminar premixed ethylene flames at a pressure of 10 bar, Combustion and Flame 100 (1995) 111-120.

[14] C.H. Kim, A.M. El-Leathy, F. Xu, G.M. Faeth, Soot surface growth and oxidation in laminar diffusion flames at pressures of 0.1-1.0 atm, Combustion and Flame 136 (2004) 191-207.

[15] R.I.A. Patterson, J. Singh, M. Balthasar, M. Kraft, W. Wagner, Extending stochastic soot simulation to higher pressures, Combustion and Flame 145 (2006) 638-642.

[16] A. Raj, M. Celnik, R. Shirley, M. Sander, R. Patterson, R. West, M. Kraft, A statistical approach to develop a detailed soot growth model using $\mathrm{PAH}$ characteristics, Combustion and Flame 156 (2009) 896-913.

[17] H. Chu, W. Han, W. Cao, M. Gu, G. Xu, Effect of methane addition to ethylene on the morphology and size distribution of soot in a laminar co-flow diffusion flame, Energy 166 (2019) 392-400.

[18] B. Shi, D. Shimokuri, S. Ishizuka, Methane/oxygen combustion in a rapidly mixed type tubular flame burner, Proceedings of the Combustion Institute 34 (2013) 3369-3377.

[19] M. Sander, R.I.A. Patterson, A. Braumann, A. Raj, M. Kraft, Developing the PAH-PP soot particle model using process informatics and uncertainty propagation, Proceedings of the Combustion Institute 33 (2011) 675-683.

[20] K.M. Leung, R.P. Lindstedt, W.P. Jones, A simplified reaction mechanism for soot formation in nonpremixed flames, Combustion and Flame 87 (1991) 289-305.

[21] D. Chen, Z. Zainuddin, E. Yapp, J. Akroyd, S. Mosbach, M. Kraft, A fully coupled simulation of $\mathrm{PAH}$ and soot growth with a population balance model, Proceedings of the Combustion Institute 34 (2013) 1827-1835.

[22] J.S. Bhatt, R.P. Lindstedt, Analysis of the impact of agglomeration and surface chemistry models on soot formation and oxidation, Proceedings of the Combustion Institute 32 (2009) 713-720.

[23] G. Blanquart, H. Pitsch, Analyzing the effects of temperature on soot formation with a joint volume-surface-hydrogen model, Combustion and Flame 156 (2009) 1614-1626. 
[24] M.S. Celnik, M. Sander, A. Raj, R.H. West, M. Kraft, Modelling soot formation in a premixed flame using an aromatic-site soot model and an improved oxidation rate, Proceedings of the Combustion Institute 32 (2009) 639-646.

[25] M. Schenk, S. Lieb, H. Vieker, A. Beyer, A. Gölzhäuser, H. Wang, K. KohseHöinghaus, Imaging nanocarbon materials: soot particles in flames are not structurally homogeneous, ChemPhysChem 14 (2013) 3248-3254.

[26] M. Frenklach, H. Wang, Detailed modeling of soot particle nucleation and growth, Symposium (International) on Combustion 23 (1991) 1559-1566.

[27] J. Appel, H. Bockhorn, M. Frenklach, Kinetic modeling of soot formation with detailed chemistry and physics: laminar premixed flames of C2 hydrocarbons, Combustion and Flame 121 (2000) 122-136.

[28] M.L. Botero, D. Chen, S. González-Calera, D. Jefferson, M. Kraft, HRTEM evaluation of soot particles produced by the non-premixed combustion of liquid fuels, Carbon 96 (2016) 459-473.

[29] J. Camacho, A.V. Singh, W. Wang, R. Shan, E.K.Y. Yapp, D. Chen, M. Kraft, H. Wang, Soot particle size distributions in premixed stretch-stabilized flat ethylene-oxygen-argon flames, Proceedings of the Combustion Institute 36 (2017) 1001-1009.

[30] M. Frenklach, New form for reduced modeling of soot oxidation: Accounting for multi-site kinetics and surface reactivity, Combustion and Flame 201 (2019) 148-159.

[31] A. Veshkini, S.B. Dworkin, M.J. Thomson, A soot particle surface reactivity model applied to a wide range of laminar ethylene/air flames, Combustion and Flame 161 (2014) 3191-3200.

[32] F. Xu, P.B. Sunderland, G.M. Faeth, Soot formation in laminar premixed ethylene/air flames at atmospheric pressure, Combustion and Flame 108 (1997) 471-493.

[33] F. Xu, K.C. Lin, G.M. Faeth, Soot formation in laminar premixed methane/oxygen flames at atmospheric pressure, Combustion and Flame 115 (1998) 195-209.

[34] M. Saffaripour, M. Kholghy, S.B. Dworkin, M.J. Thomson, A numerical and experimental study of soot formation in a laminar coflow diffusion flame of a Jet A-1 surrogate, Proceedings of the Combustion Institute 34 (2013) 1057-1065.

[35] M. Kholghy, M. Saffaripour, C. Yip, M.J. Thomson, The evolution of soot morphology in a laminar coflow diffusion flame of a surrogate for Jet A-1, Combustion and Flame 160 (2013) 2119-2130.

[36] Y. Wang, S.H. Chung, Soot formation in laminar counterflow flames, Progress in Energy and Combustion Science 74 (2019) 152-238.

[37] M.R. Kholghy, A. Veshkini, M.J. Thomson, The core-shell internal nanostructure of soot - A criterion to model soot maturity, Carbon 100 (2016) 508536.

[38] Z. Hong, D. Davidson, E. Barbour, R. Hanson, A new shock tube study of the $\mathrm{H}+\mathrm{O} 2 \rightarrow \mathrm{OH}+\mathrm{O}$ reaction rate using tunable diode laser absorption of $\mathrm{H} 2 \mathrm{O}$ near $2.5 \mu \mathrm{m}$, Proceedings of the Combustion Institute 33 (2011) 309-316. 
[39] H. Wang, D.A. Sheen, Combustion kinetic model uncertainty quantification, propagation and minimization, Progress in Energy and Combustion Science 47 (2015) 1-31.

[40] R.A. Dobbins, Hydrocarbon nanoparticles formed in flames and diesel engines, Aerosol Science and Technology 41 (2007) 485-496.

[41] T.S. Totton, D. Chakrabarti, A.J. Misquitta, M. Sander, D.J. Wales, M. Kraft, Modelling the internal structure of nascent soot particles, Combustion and Flame 157 (2010) 909-914.

[42] D. Chen, J. Akroyd, S. Mosbach, D. Opalka, M. Kraft, Solid-liquid transitions in homogenous ovalene, hexabenzocoronene and circumcoronene clusters: A molecular dynamics study, Combustion and Flame 162 (2015) 486-495.

[43] C. Liu, A.V. Singh, C. Saggese, Q. Tang, D. Chen, K. Wan, M. Vinciguerra, M. Commodo, G. De Falco, P. Minutolo, A. D’Anna, H. Wang, Flame-formed carbon nanoparticles exhibit quantum dot behaviors, doi:10.1073/pnas.1900205116 \%J Proceedings of the National Academy of Sciences(2019) 201900205.

[44] D. Chen, T.S. Totton, J.W.J. Akroyd, S. Mosbach, M. Kraft, Size-dependent melting of polycyclic aromatic hydrocarbon nano-clusters: A molecular dynamics study, Carbon 67 (2014) 79-91.

[45] D. Chen, T.S. Totton, J. Akroyd, S. Mosbach, M. Kraft, Phase change of polycyclic aromatic hydrocarbon clusters by mass addition, Carbon 77 (2014) 2535.

[46] D. Chen, J. Akroyd, S. Mosbach, M. Kraft, Surface reactivity of polycyclic aromatic hydrocarbon clusters, Proceedings of the Combustion Institute 35 (2015) 1811-1818.

[47] M.F. Sanner, A.J. Olson, J.C. Spehner, Reduced surface: an efficient way to compute molecular surfaces, Biopolymers 38 (1996) 305-320.

[48] A. Bondi, van der Waals Volumes and Radii, The Journal of Physical Chemistry 68 (1964) 441-451.

[49] K. Wan, D. Chen, H. Wang, On imaging nascent soot by transmission electron microscopy, Combustion and Flame 198 (2018) 260-266.

[50] A.M. Nienow, J.T. Roberts, M.R. Zachariah, Surface chemistry of nanometersized aerosol particles: reactions of molecular oxygen with $30 \mathrm{~nm}$ soot particles as a function of oxygen partial pressure, The Journal of Physical Chemistry B 109 (2005) 5561-5568.

[51] J.P. Cain, J. Camacho, D.J. Phares, H. Wang, A. Laskin, Evidence of aliphatics in nascent soot particles in premixed ethylene flames, Proceedings of the Combustion Institute 33 (2011) 533-540.

[52] D. Chen, H. Wang, HOMO-LUMO energy splitting in polycyclic aromatic hydrocarbons and their derivatives, Proceedings of the Combustion Institute 37 (2019) 953-959.

[53] M.R. Kholghy, G.A. Kelesidis, S.E. Pratsinis, Reactive polycyclic aromatic hydrocarbon dimerization drives soot nucleation, Physical Chemistry Chemical Physics 20 (2018) 10926-10938. 
[54] H. Yuan, W. Kong, F. Liu, D. Chen, Study on soot nucleation and growth from PAHs and some reactive species at flame temperatures by ReaxFF molecular dynamics, Chemical Engineering Science 195 (2019) 748-757.

[55] K.O. Johansson, M.P. Head-Gordon, P.E. Schrader, K.R. Wilson, H.A. Michelsen, Resonance-stabilized hydrocarbon-radical chain reactions may explain soot inception and growth, 361 (2018) 997-1000.

[56] F. Schulz, M. Commodo, K. Kaiser, G. De Falco, P. Minutolo, G. Meyer, A. D`Anna, L. Gross, Insights into incipient soot formation by atomic force microscopy, Proceedings of the Combustion Institute 37 (2019) 885-892.

[57] K. Bowal, J.W. Martin, A.J. Misquitta, M. Kraft, Ion-induced soot nucleation using a new potential for curved aromatics, Combustion Science and Technology 191 (2019) 747-765.

[58] J.W. Martin, A. Menon, C.T. Lao, J. Akroyd, M. Kraft, Dynamic polarity of curved aromatic soot precursors, Combustion and Flame 206 (2019) 150-157.

[59] Q. Zhang, H. Guo, F. Liu, G. Smallwood, M. Thomson, Modeling of soot aggregate formation and size distribution in a laminar ethylene/air coflow diffusion flame with detailed PAH chemistry and an advanced sectional aerosol dynamics model, Proceedings of the Combustion Institute 32 (2009) 761-768.

[60] F. Liu, S. Dworkin, M. Thomson, G. Smallwood, Modeling DME addition effects to fuel on $\mathrm{PAH}$ and soot in laminar coflow ethylene/air diffusion flames using two PAH mechanisms, Combustion Science and Technology 184 (2012) 966979.

[61] M. Saffaripour, P. Zabeti, S. Dworkin, Q. Zhang, H. Guo, F. Liu, G. Smallwood, M. Thomson, A numerical and experimental study of a laminar sooting coflow Jet-A1 diffusion flame, Proceedings of the Combustion Institute 33 (2011) 601-608.

[62] A.M. El-Leathy, C.H. Kim, G.M. Faeth, F. Xu, Soot surface reactions in hightemperature laminar diffusion flames, AIAA Journal 42 (2004) 988-996.

[63] F. Zhao, W. Yang, D. Zhou, W. Yu, J. Li, K.L. Tay, Numerical modelling of soot formation and oxidation using phenomenological soot modelling approach in a dual-fueled compression ignition engine, Fuel 188 (2017) 382-389.

[64] J. Singh, M. Balthasar, M. Kraft, W. Wagner, Stochastic modeling of soot particle size and age distributions in laminar premixed flames, Proceedings of the Combustion Institute 30 (2005) 1457-1465.

[65] H. Guo, F. Liu, G.J. Smallwood, Ö.L. Gülder, Numerical study on the influence of hydrogen addition on soot formation in a laminar ethylene-air diffusion flame, Combustion and Flame 145 (2006) 324-338.

[66] S.B. Dworkin, Q. Zhang, M.J. Thomson, N.A. Slavinskaya, U. Riedel, Application of an enhanced PAH growth model to soot formation in a laminar coflow ethylene/air diffusion flame, Combustion and Flame 158 (2011) 1682-1695. [67] Y. Wang, A. Raj, S.H. Chung, Soot modeling of counterflow diffusion flames of ethylene-based binary mixture fuels, Combustion and Flame 162 (2015) 586596. 
[68] A. Khosousi, S.B. Dworkin, Soot surface reactivity during surface growth and oxidation in laminar diffusion flames, Combustion and Flame 162 (2015) 45234532.

[69] A. Khosousi, S.B. Dworkin, Detailed modelling of soot oxidation by O2 and $\mathrm{OH}$ in laminar diffusion flames, Proceedings of the Combustion Institute 35 (2015) 1903-1910. 